\title{
VANISHING COEFFICIENTS IN SOME $q$-SERIES EXPANSIONS
}

\author{
DAZHAO TANG
}

\begin{abstract}
Motivated by the recent work of Hirschhorn on vanishing coefficients of the arithmetic progressions in certain $q$-series expansions, we study some variants of these $q$-series and prove some comparable results. For instance, let

$$
\left(-q,-q^{4} ; q^{5}\right)_{\infty}^{2}\left(q^{4}, q^{6} ; q^{10}\right)_{\infty}=\sum_{n=0}^{\infty} a_{1}(n) q^{n}
$$
\end{abstract}

then

$$
a_{1}(5 n+3)=0
$$

\section{INTRODUCTION}

The study of vanishing coefficients in infinite product expansions, which was derived from the Hardy-Ramanujan-Rademacher expansions for quotients of certain infinite products, can be traced back to Richmond and Szekeres [9]. Soon after, Andrews and Bressoud [2] proved a general theorem for certain Rogers-Ramanujan type infinite products, which contains the results of Richmond and Szekeres as special cases. Later, Alladi and Gordon [1] arrived at a more general result with some restrictions. In a recent paper, among other things, McLaughlin [8] further generalized the results of Alladi and Gordon.

Quite recently, Hirschhorn [5] studied the following two $q$-series:

$$
\begin{gathered}
\left(-q,-q^{4} ; q^{5}\right)_{\infty}\left(q, q^{9} ; q^{10}\right)_{\infty}^{3}=\sum_{n=0}^{\infty} a(n) q^{n} \\
\left(-q^{2},-q^{3} ; q^{5}\right)_{\infty}\left(q^{3}, q^{7} ; q^{10}\right)_{\infty}^{3}=\sum_{n=0}^{\infty} b(n) q^{n} .
\end{gathered}
$$

He proved that

$$
\begin{aligned}
& a(5 n+2)=a(5 n+4)=0 \\
& b(5 n+1)=b(5 n+4)=0 .
\end{aligned}
$$

Date: December 25, 2019.

2010 Mathematics Subject Classification. 11F33, 30B10.

Key words and phrases. Vanishing coefficients; $q$-series expansions; Jacobi's triple product identity. 
Here and in what follows, we adopt the following customary $q$-series notations:

$$
\begin{aligned}
(a ; q)_{\infty} & :=\prod_{n=0}^{\infty}\left(1-a q^{n}\right), \\
\left(a_{1}, a_{2}, \cdots, a_{m} ; q\right)_{\infty} & :=\left(a_{1} ; q\right)_{\infty}\left(a_{2} ; q\right)_{\infty} \cdots\left(a_{m} ; q\right)_{\infty}, \quad \text { for } \quad|q|<1 .
\end{aligned}
$$

In this paper, we consider some variants of (1.1) and (1.2), and obtain some comparable results on vanishing coefficients in these $q$-series expansions.

Define

$$
\begin{aligned}
\left(-q,-q^{4} ; q^{5}\right)_{\infty}^{2}\left(q^{4}, q^{6} ; q^{10}\right)_{\infty} & =\sum_{n=0}^{\infty} a_{1}(n) q^{n}, \\
\left(-q^{2},-q^{3} ; q^{5}\right)_{\infty}^{2}\left(q^{2}, q^{8} ; q^{10}\right)_{\infty} & =\sum_{n=0}^{\infty} b_{1}(n) q^{n} \\
\left(-q,-q^{4} ; q^{5}\right)_{\infty}^{3}\left(q^{2}, q^{8} ; q^{10}\right)_{\infty} & =\sum_{n=0}^{\infty} a_{2}(n) q^{n}, \\
\left(-q^{2},-q^{3} ; q^{5}\right)_{\infty}^{3}\left(q^{4}, q^{6} ; q^{10}\right)_{\infty} & =\sum_{n=0}^{\infty} b_{2}(n) q^{n}, \\
\left(-q,-q^{4} ; q^{5}\right)_{\infty}^{3}\left(q^{3}, q^{7} ; q^{10}\right)_{\infty} & =\sum_{n=0}^{\infty} a_{3}(n) q^{n}, \\
\left(-q^{2},-q^{3} ; q^{5}\right)_{\infty}^{3}\left(q, q^{9} ; q^{10}\right)_{\infty} & =\sum_{n=0}^{\infty} b_{3}(n) q^{n} .
\end{aligned}
$$

Theorem 1.1. For any integer $n \geq 0$,

$$
\begin{aligned}
& a_{1}(5 n+3)=0, \\
& b_{1}(5 n+1)=0, \\
& a_{2}(5 n+4)=0, \\
& b_{2}(5 n+1)=0, \\
& a_{3}(5 n+3)=a_{3}(5 n+4)=0, \\
& b_{3}(5 n+3)=b_{3}(5 n+4)=0 .
\end{aligned}
$$

\section{ProOfs}

Ramanujan's theta function is defined by

$$
f(a, b):=\sum_{n=-\infty}^{\infty} a^{n(n+1) / 2} b^{n(n-1) / 2},
$$


where $|a b|<1$. The function $f(a, b)$ enjoys the well-known Jacobi triple product identity [4, p. 35, Entry 19]:

$$
f(a, b)=(-a,-b, a b ; a b)_{\infty} .
$$

For notational convenience, denote

$$
E_{j}:=\left(q^{j} ; q^{j}\right)_{\infty}
$$

Let $k, l$ be positive integers and $G(q)=\sum_{n=0}^{\infty} g(n) q^{n}$ be a formal power series. Define an operator $H_{k, l}$ by

$$
H_{k, l}(G(q)):=\sum_{n=0}^{\infty} g(k n+l) q^{k n+l} .
$$

Recall that Ramanujan's classical theta functions $\varphi(q)$ and $\psi(q)$ are given by [7, Eqs. (1.5.4) and (1.5.5)]:

$$
\begin{aligned}
& \varphi(q):=f(q, q)=\sum_{n=-\infty}^{\infty} q^{n^{2}}=\frac{E_{2}^{5}}{E_{1}^{2} E_{4}^{2}}, \\
& \psi(q):=f\left(q, q^{3}\right)=\sum_{n=0}^{\infty} q^{n(n+1) / 2}=\frac{E_{2}^{2}}{E_{1}} .
\end{aligned}
$$

Lemma 2.1. Define

$$
\begin{aligned}
& S_{1}=\sum_{m, n=-\infty}^{\infty} q^{20 m^{2}+2 m+20 n^{2}+6 n}, \quad S_{2}=\sum_{m, n=-\infty}^{\infty} q^{20 m^{2}+18 m+20 n^{2}+6 n}, \\
& S_{3}=\sum_{m, n=-\infty}^{\infty} q^{20 m^{2}+2 m+20 n^{2}+14 n}, \quad S_{4}=\sum_{m, n=-\infty}^{\infty} q^{20 m^{2}+18 m+20 n^{2}+14 n}, \\
& S_{5}=\sum_{m, n=-\infty}^{\infty} q^{20 m^{2}+2 m+20 n^{2}+4 n}, \quad S_{6}=\sum_{m, n=-\infty}^{\infty} q^{20 m^{2}+18 m+20 n^{2}+4 n} \\
& S_{7}=\sum_{m, n=-\infty}^{\infty} q^{20 m^{2}+2 m+20 n^{2}+16 n}, \quad S_{8}=\sum_{m, n=-\infty}^{\infty} q^{20 m^{2}+18 m+20 n^{2}+16 n} .
\end{aligned}
$$

Then

$$
H_{5,3}\left(S_{1}-q^{4} S_{2}\right)=H_{5,3}\left(q^{2} S_{3}-q^{6} S_{4}\right)=H_{5,3}\left(q S_{5}-q^{5} S_{6}\right)=H_{5,3}\left(q^{4} S_{7}-q^{8} S_{8}\right)=0 .
$$

Proof. We only prove $H_{5,3}\left(S_{1}-q^{4} S_{2}\right)=0$, and the remaining cases are similar.

In $S_{1}$, if $2 m+6 n \equiv 3(\bmod 5)$, then $2 m+n \equiv-2(\bmod 5)$. Of course, $m-2 n \equiv-1$ $(\bmod 5)$. Assume $2 m+n=5 r-2$ and $m-2 n=-5 s-1$, it follows that $m=2 r-s-1$ 
and $n=r+2 s$. Thus $H_{5,3}\left(S_{1}\right)$ equals

$$
\begin{aligned}
& \sum_{r, s=-\infty}^{\infty} q^{20(2 r-s-1)^{2}+2(2 r-s-1)+20(r+2 s)^{2}+6(r+2 s)} \\
= & q^{18} \sum_{r, s=-\infty}^{\infty} q^{100 r^{2}+100 s^{2}-70 r+50 s}=q^{18} \sum_{r, s=-\infty}^{\infty} q^{100 r^{2}+100 s^{2}+70 r+50 s} .
\end{aligned}
$$

In $S_{2}, 18 m+6 n \equiv-1,-2 m+n \equiv-1$, that is, $2 m-n \equiv 1, m+2 n \equiv-2$. Let $2 m-n=5 r+1$ and $m+2 n=-5 s-2$, then $m=2 r-s, n=-r-2 s-1$. Therefore, $H_{5,3}\left(q^{4} S_{2}\right)$ is

$$
\begin{aligned}
& q^{4} \sum_{r, s=-\infty}^{\infty} q^{20(2 r-s)^{2}+18(2 r-s)+20(-r-2 s-1)^{2}+6(-r-2 s-1)} \\
& =q^{18} \sum_{r, s=-\infty}^{\infty} q^{100 r^{2}+100 s^{2}+70 r+50 s}
\end{aligned}
$$

as desired.

The above $q$-series manipulation was developed by Hirschhorn [5], therefore we called it Hirschhorn's operation in the sequel.

We have

$$
\begin{aligned}
\left(-q,-q^{4} ; q^{5}\right)_{\infty}^{2}=\frac{f\left(q, q^{4}\right)^{2}}{E_{5}^{2}}= & \frac{1}{E_{5}^{2}} \sum_{m, n=-\infty}^{\infty} q^{\left(5 m^{2}+3 m+5 n^{2}+3 n\right) / 2} \\
= & \frac{1}{E_{5}^{2}}\left(\sum_{r, s=-\infty}^{\infty} q^{\left(5(r+s)^{2}+3(r+s)+5(r-s)^{2}+3(r-s)\right) / 2}\right. \\
& \left.+\sum_{r, s=-\infty}^{\infty} q^{\left(5(r+s-1)^{2}+3(r+s-1)+5(r-s)^{2}+3(r-s)\right) / 2}\right) \\
= & \frac{E_{10}^{5}}{E_{5}^{4} E_{20}^{2}} \sum_{n=-\infty}^{\infty} q^{5 n^{2}+3 n}+2 q \frac{E_{20}^{2}}{E_{5}^{2} E_{10}} \sum_{n=-\infty}^{\infty} q^{5 n^{2}+2 n} \\
= & \frac{E_{10}^{5}}{E_{5}^{4} E_{20}^{2}}\left(\sum_{n=-\infty}^{\infty} q^{20 n^{2}+6 n}+q^{2} \sum_{n=-\infty}^{\infty} q^{20 n^{2}+14 n}\right) \\
& +2 \frac{E_{20}^{2}}{E_{5}^{2} E_{10}}\left(q \sum_{n=-\infty}^{\infty} q^{20 n^{2}+4 n}+q^{4} \sum_{n=-\infty}^{\infty} q^{20 n^{2}+16 n}\right) .
\end{aligned}
$$

Moreover,

$$
\left(q^{4}, q^{6} ; q^{10}\right)_{\infty}=\frac{1}{E_{10}} \sum_{m=-\infty}^{\infty}(-1)^{m} q^{5 m^{2}+m}
$$




$$
=\frac{1}{E_{10}}\left(\sum_{m=-\infty}^{\infty} q^{20 m^{2}+2 m}-q^{4} \sum_{m=-\infty}^{\infty} q^{20 m^{2}+18 m}\right) .
$$

We then get

$$
\begin{aligned}
\sum_{n=0}^{\infty} a_{1}(n) q^{n}= & \frac{1}{E_{10}}\left(\sum_{m=-\infty}^{\infty} q^{20 m^{2}+2 m}-q^{4} \sum_{m=-\infty}^{\infty} q^{20 m^{2}+18 m}\right) \\
& \times\left(\frac{E_{10}^{5}}{E_{5}^{4} E_{20}^{2}} \sum_{n=-\infty}^{\infty} q^{20 n^{2}+6 n}+q^{2} \frac{E_{10}^{5}}{E_{5}^{4} E_{20}^{2}} \sum_{n=-\infty}^{\infty} q^{20 n^{2}+14 n}\right. \\
& \left.+2 q \frac{E_{20}^{2}}{E_{5}^{2} E_{10}} \sum_{n=-\infty}^{\infty} q^{20 n^{2}+4 n}+2 q^{4} \frac{E_{20}^{2}}{E_{5}^{2} E_{10}} \sum_{n=-\infty}^{\infty} q^{20 n^{2}+16 n}\right) \\
= & \frac{E_{10}^{4}}{E_{5}^{4} E_{20}^{2}}\left(S_{1}-q^{4} S_{2}+q^{2} S_{3}-q^{6} S_{4}\right)+\frac{2 E_{20}^{2}}{E_{5}^{2} E_{10}^{2}}\left(q S_{5}-q^{5} S_{6}+q^{4} S_{7}-q^{8} S_{8}\right) .
\end{aligned}
$$

In view of Lemma 2.1, we obtain (1.3).

The proof of (1.4) is similar so is omitted here.

Now, we are ready to prove (1.5)-(1.8).

We start with

$$
\begin{aligned}
& \sum_{n=0}^{\infty} a_{2}(n) q^{n}=\frac{f\left(q, q^{4}\right)}{E_{5} E_{10}}\left(\sum_{m=-\infty}^{\infty} q^{20 m^{2}+6 m}-q^{2} \sum_{m=-\infty}^{\infty} q^{20 m^{2}+14 m}\right) \\
& \times\left(\frac{E_{10}^{5}}{E_{5}^{4} E_{20}^{2}} \sum_{n=-\infty}^{\infty} q^{20 n^{2}+6 n}+q^{2} \frac{E_{10}^{5}}{E_{5}^{4} E_{20}^{2}} \sum_{n=-\infty}^{\infty} q^{20 n^{2}+14 n}\right. \\
& \left.+2 q \frac{E_{20}^{2}}{E_{5}^{2} E_{10}} \sum_{n=-\infty}^{\infty} q^{20 n^{2}+4 n}+2 q^{4} \frac{E_{20}^{2}}{E_{5}^{2} E_{10}} \sum_{n=-\infty}^{\infty} q^{20 n^{2}+16 n}\right) \\
& =\frac{E_{10}^{4}}{E_{5}^{5} E_{20}^{2}} f\left(q, q^{4}\right)\left(\sum_{r, s=-\infty}^{\infty} q^{20(r+s)^{2}+6(r+s)+20(r-s)^{2}+6(r-s)}\right. \\
& +\sum_{r, s=-\infty}^{\infty} q^{20(r+s-1)^{2}+6(r+s-1)+20(r-s)^{2}+6(r-s)} \\
& -q^{4} \sum_{r, s=-\infty}^{\infty} q^{20(r+s)^{2}+14(r+s)+20(r-s)^{2}+14(r-s)} \\
& \left.-q^{4} \sum_{r, s=-\infty}^{\infty} q^{20(r+s-1)^{2}+14(r+s-1)+20(r-s)^{2}+14(r-s)}\right)
\end{aligned}
$$




$$
\begin{aligned}
& +\frac{2 E_{20}^{2}}{E_{5}^{3} E_{10}^{2}} f\left(q, q^{4}\right)\left(q \sum_{r, s=-\infty}^{\infty} q^{20(r+s)^{2}+6(r+s)+20(r-s)^{2}+4(r-s)}\right. \\
& +q \sum_{r, s=-\infty}^{\infty} q^{20(r+s-1)^{2}+6(r+s-1)+20(r-s)^{2}+4(r-s)} \\
& +q^{4} \sum_{r, s=-\infty}^{\infty} q^{20(r+s)^{2}+6(r+s)+20(r-s)^{2}+16(r-s)} \\
& +q^{4} \sum_{r, s=-\infty}^{\infty} q^{20(r+s-1)^{2}+6(r+s-1)+20(r-s)^{2}+16(r-s)} \\
& -q^{3} \sum_{r, s=-\infty}^{\infty} q^{20(r+s)^{2}+14(r+s)+20(r-s)^{2}+4(r-s)} \\
& -q^{3} \sum_{r, s=-\infty}^{\infty} q^{20(r+s-1)^{2}+14(r+s-1)+20(r-s)^{2}+4(r-s)} \\
& -q^{6} \sum_{r, s=-\infty}^{\infty} q^{20(r+s)^{2}+14(r+s)+20(r-s)^{2}+16(r-s)} \\
& \left.-q^{6} \sum_{r, s=-\infty}^{\infty} q^{20(r+s-1)^{2}+14(r+s-1)+20(r-s)^{2}+16(r-s)}\right) \\
& =\frac{E_{10}^{4} E_{80}^{5} f\left(q, q^{4}\right)}{E_{5}^{5} E_{20}^{2} E_{40}^{2} E_{160}^{2}}\left(\sum_{n=-\infty}^{\infty} q^{40 n^{2}+12 n}-q^{4} \sum_{n=-\infty}^{\infty} q^{40 n^{2}+28 n}\right) \\
& +\frac{2 E_{10}^{4} E_{160}^{2} f\left(q, q^{4}\right)}{E_{5}^{5} E_{20}^{2} E_{80}}\left(q^{14} \sum_{n=-\infty}^{\infty} q^{40 n^{2}+28 n}-q^{10} \sum_{n=-\infty}^{\infty} q^{40 n^{2}+12 n}\right) \\
& +\frac{2 E_{20}^{2} f\left(q^{30}, q^{50}\right) f\left(q, q^{4}\right)}{E_{5}^{3} E_{10}^{2}}\left(q \sum_{n=-\infty}^{\infty} q^{40 n^{2}+2 n}-q^{10} \sum_{n=-\infty}^{\infty} q^{40 n^{2}+38 n}\right. \\
& \left.+q^{4} \sum_{n=-\infty}^{\infty} q^{40 n^{2}+22 n}-q^{3} \sum_{n=-\infty}^{\infty} q^{40 n^{2}+18 n}\right) \\
& +\frac{2 E_{20}^{2} f\left(q^{10}, q^{70}\right) f\left(q, q^{4}\right)}{E_{5}^{3} E_{10}^{2}}\left(q^{15} \sum_{n=-\infty}^{\infty} q^{40 n^{2}+38 n}-q^{6} \sum_{n=-\infty}^{\infty} q^{40 n^{2}+2 n}\right. \\
& \left.+q^{8} \sum_{n=-\infty}^{\infty} q^{40 n^{2}+18 n}-q^{9} \sum_{n=-\infty}^{\infty} q^{40 n^{2}+22 n}\right) \text {. }
\end{aligned}
$$


Denote

$$
\begin{aligned}
& A_{1}:=f\left(q, q^{4}\right) \sum_{n=-\infty}^{\infty} q^{40 n^{2}+12 n}-q^{4} f\left(q, q^{4}\right) \sum_{n=-\infty}^{\infty} q^{40 n^{2}+28 n} \\
& A_{2}:=q^{14} f\left(q, q^{4}\right) \sum_{n=-\infty}^{\infty} q^{40 n^{2}+28 n}-q^{10} f\left(q, q^{4}\right) \sum_{n=-\infty}^{\infty} q^{40 n^{2}+12 n} \\
& A_{3}:=q f\left(q, q^{4}\right) \sum_{n=-\infty}^{\infty} q^{40 n^{2}+2 n}-q^{10} f\left(q, q^{4}\right) \sum_{n=-\infty}^{\infty} q^{40 n^{2}+38 n} \\
& A_{4}:=q^{4} f\left(q, q^{4}\right) \sum_{n=-\infty}^{\infty} q^{40 n^{2}+22 n}-q^{3} f\left(q, q^{4}\right) \sum_{n=-\infty}^{\infty} q^{40 n^{2}+18 n} \\
& A_{5}:=q^{15} f\left(q, q^{4}\right) \sum_{n=-\infty}^{\infty} q^{40 n^{2}+38 n}-q^{6} f\left(q, q^{4}\right) \sum_{n=-\infty}^{\infty} q^{40 n^{2}+2 n} \\
& A_{6}:=q^{8} f\left(q, q^{4}\right) \sum_{n=-\infty}^{\infty} q^{40 n^{2}+18 n}-q^{9} f\left(q, q^{4}\right) \sum_{n=-\infty}^{\infty} q^{40 n^{2}+22 n} .
\end{aligned}
$$

Next, we prove that

$$
H_{5,4}\left(A_{i}\right)=0, \quad \text { for } \quad 1 \leq i \leq 6 .
$$

We only prove the case $A_{1}$ here because the proofs of remaining cases are similar.

Also,

$$
\begin{aligned}
f\left(q, q^{4}\right) & =\sum_{m=-\infty}^{\infty} q^{\left(5 m^{2}+3 m\right) / 2} \\
& =\sum_{m=-\infty}^{\infty} q^{10 m^{2}+3 m}+q \sum_{m=-\infty}^{\infty} q^{10 m^{2}+7 m} \\
& =\sum_{m=-\infty}^{\infty} q^{40 m^{2}+6 m}+q^{7} \sum_{m=-\infty}^{\infty} q^{40 m^{2}+34 m}+q \sum_{m=-\infty}^{\infty} q^{40 m^{2}+14 m}+q^{4} \sum_{m=-\infty}^{\infty} q^{40 m^{2}+26 m} .
\end{aligned}
$$

Therefore,

$$
A_{1}=P_{1}-P_{2}+P_{3}-P_{4}+P_{5}-P_{6}+P_{7}-P_{8}
$$

where

$$
\begin{aligned}
& P_{1}=\sum_{m, n=-\infty}^{\infty} q^{40 m^{2}+6 m+40 n^{2}+12 n}, \quad P_{2}=q^{8} \sum_{m, n=-\infty}^{\infty} q^{40 m^{2}+26 m+40 n^{2}+28 n}, \\
& P_{3}=q \sum_{m, n=-\infty}^{\infty} q^{40 m^{2}+14 m+40 n^{2}+12 n}, \quad P_{4}=q^{11} \sum_{m, n=-\infty}^{\infty} q^{40 m^{2}+34 m+40 n^{2}+28 n},
\end{aligned}
$$




$$
\begin{array}{ll}
P_{5}=q^{4} \sum_{m, n=-\infty}^{\infty} q^{40 m^{2}+26 m+40 n^{2}+12 n}, & P_{6}=q^{4} \sum_{m, n=-\infty}^{\infty} q^{40 m^{2}+6 m+40 n^{2}+28 n}, \\
P_{7}=q^{7} \sum_{m, n=-\infty}^{\infty} q^{40 m^{2}+34 m+40 n^{2}+12 n}, & P_{8}=q^{5} \sum_{m, n=-\infty}^{\infty} q^{40 m^{2}+14 m+40 n^{2}+28 n} .
\end{array}
$$

By Hirschhorn's operation, we have

$$
H_{5,4}\left(P_{2 i-1}-P_{2 i}\right)=0, \quad \text { for } \quad 1 \leq i \leq 4 .
$$

This proves (1.5).

Similarly, we get

$$
\begin{aligned}
& \sum_{n=0}^{\infty} a_{3}(n) q^{n}=\frac{f\left(q, q^{4}\right)}{E_{5}^{3} E_{10}}\left(\sum_{m=-\infty}^{\infty} q^{20 m^{2}+4 m}-q^{3} \sum_{m=-\infty}^{\infty} q^{20 m^{2}+16 m}\right) \\
& \times\left(\frac{E_{10}^{5}}{E_{5}^{2} E_{20}^{2}} \sum_{n=-\infty}^{\infty} q^{20 n^{2}+6 n}+q^{2} \frac{E_{10}^{5}}{E_{5}^{2} E_{20}^{2}} \sum_{n=-\infty}^{\infty} q^{20 n^{2}+14 n}\right. \\
& \left.+2 q \frac{E_{20}^{2}}{E_{10}} \sum_{n=-\infty}^{\infty} q^{20 n^{2}+4 n}+2 q^{4} \frac{E_{20}^{2}}{E_{10}} \sum_{n=-\infty}^{\infty} q^{20 n^{2}+16 n}\right) \\
& =\frac{E_{10}^{4} f\left(q^{30}, q^{50}\right) f\left(q, q^{4}\right)}{E_{5}^{5} E_{20}^{2}}\left(\sum_{n=-\infty}^{\infty} q^{40 n^{2}+2 n}+q^{2} \sum_{n=-\infty}^{\infty} q^{40 n^{2}+18 n}\right. \\
& \left.-q^{3} \sum_{n=\infty}^{\infty} q^{40 n^{2}+22 n}-q^{9} \sum_{n=\infty}^{\infty} q^{40 n^{2}+38 n}\right) \\
& +\frac{E_{10}^{4} f\left(q^{10}, q^{70}\right) f\left(q, q^{4}\right)}{E_{5}^{5} E_{20}^{2}}\left(q^{8} \sum_{n=-\infty}^{\infty} q^{40 n^{2}+22 n}+q^{14} \sum_{n=-\infty}^{\infty} q^{40 n^{2}+38 n}\right. \\
& \left.-q^{7} \sum_{n=\infty}^{\infty} q^{40 n^{2}+18 n}-q^{5} \sum_{n=\infty}^{\infty} q^{40 n^{2}+2 n}\right) \\
& +\frac{2 E_{20}^{2} E_{80}^{5} f\left(q, q^{4}\right)}{E_{5}^{3} E_{10}^{2} E_{40}^{2} E_{160}^{2}}\left(q \sum_{n=-\infty}^{\infty} q^{40 n^{2}+8 n}-q^{7} \sum_{n=-\infty}^{\infty} q^{40 n^{2}+32 n}\right) \\
& +\frac{4 E_{20}^{2} E_{160}^{2} f\left(q, q^{4}\right)}{E_{5}^{3} E_{10}^{2} E_{80}}\left(q^{17} \sum_{n=-\infty}^{\infty} q^{40 n^{2}+32 n}-q^{11} \sum_{n=-\infty}^{\infty} q^{40 n^{2}+8 n}\right) .
\end{aligned}
$$

Now, we discuss the following two cases:

1) Denote

$$
B_{1}:=f\left(q, q^{4}\right) \sum_{m=-\infty}^{\infty} q^{20 n^{2}+2 n}-q^{9} f\left(q, q^{4}\right) \sum_{n=\infty}^{\infty} q^{40 n^{2}+38 n}
$$




$$
\begin{aligned}
& B_{2}:=q^{2} f\left(q, q^{4}\right) \sum_{n=-\infty}^{\infty} q^{40 n^{2}+18 n}-q^{3} f\left(q, q^{4}\right) \sum_{n=\infty}^{\infty} q^{40 n^{2}+22 n}, \\
& B_{3}:=q^{8} f\left(q, q^{4}\right) \sum_{n=-\infty}^{\infty} q^{40 n^{2}+22 n}-q^{7} f\left(q, q^{4}\right) \sum_{n=\infty}^{\infty} q^{40 n^{2}+18 n}, \\
& B_{4}:=q^{14} f\left(q, q^{4}\right) \sum_{n=-\infty}^{\infty} q^{40 n^{2}+38 n}-q^{5} f\left(q, q^{4}\right) \sum_{n=\infty}^{\infty} q^{40 n^{2}+2 n}, \\
& B_{5}:=q f\left(q, q^{4}\right) \sum_{n=-\infty}^{\infty} q^{40 n^{2}+8 n}-q^{7} f\left(q, q^{4}\right) \sum_{n=-\infty}^{\infty} q^{40 n^{2}+32 n}, \\
& B_{6}:=q^{17} f\left(q, q^{4}\right) \sum_{n=-\infty}^{\infty} q^{40 n^{2}+32 n}-q^{11} f\left(q, q^{4}\right) \sum_{n=-\infty}^{\infty} q^{40 n^{2}+8 n} .
\end{aligned}
$$

By reasoning as above, we obtain

$$
H_{5,3}\left(B_{i}\right)=0, \quad \text { for } \quad 1 \leq i \leq 6 .
$$

Therefore,

$$
a_{3}(5 n+3)=0 .
$$

2) Denote

$$
\begin{aligned}
& C_{1}:=f\left(q, q^{4}\right) \sum_{m=-\infty}^{\infty} q^{20 n^{2}+2 n}-q^{3} f\left(q, q^{4}\right) \sum_{n=\infty}^{\infty} q^{40 n^{2}+22 n}, \\
& C_{2}:=q^{2} f\left(q, q^{4}\right) \sum_{n=-\infty}^{\infty} q^{40 n^{2}+18 n}-q^{9} f\left(q, q^{4}\right) \sum_{n=\infty}^{\infty} q^{40 n^{2}+38 n}, \\
& C_{3}:=q^{8} f\left(q, q^{4}\right) \sum_{n=-\infty}^{\infty} q^{40 n^{2}+22 n}-q^{5} f\left(q, q^{4}\right) \sum_{n=\infty}^{\infty} q^{40 n^{2}+2 n}, \\
& C_{4}:=q^{14} f\left(q, q^{4}\right) \sum_{n=-\infty}^{\infty} q^{40 n^{2}+38 n}-q^{7} f\left(q, q^{4}\right) \sum_{n=\infty}^{\infty} q^{40 n^{2}+18 n}, \\
& C_{5}:=q f\left(q, q^{4}\right) \sum_{n=-\infty}^{\infty} q^{40 n^{2}+8 n}-q^{7} f\left(q, q^{4}\right) \sum_{n=-\infty}^{\infty} q^{40 n^{2}+32 n}, \\
& C_{6}:=q^{17} f\left(q, q^{4}\right) \sum_{n=-\infty}^{\infty} q^{40 n^{2}+32 n}-q^{11} f\left(q, q^{4}\right) \sum_{n=-\infty}^{\infty} q^{40 n^{2}+8 n} .
\end{aligned}
$$

Thus, as above,

$$
H_{5,4}\left(C_{i}\right)=0, \quad \text { for } \quad 1 \leq i \leq 6 .
$$


Furthermore,

$$
a_{3}(5 n+4)=0
$$

This establishes (1.7).

The proofs of (1.6) and (1.8) are similar to those of (1.5) and (1.7).

\section{FinAl REMARKS}

On one hand, there are more analogous results on vanishing coefficients in other infinite product expansions beyond this paper. Define

$$
\begin{aligned}
& \left(-q^{r},-q^{t-r} ; q^{t}\right)_{\infty}^{3}\left(q^{s}, q^{2 t-s} ; q^{2 t}\right)_{\infty}:=\sum_{n=0}^{\infty} a_{r, s, t}(n) q^{n} \\
& \left(-q^{r},-q^{t-r} ; q^{t}\right)_{\infty}\left(q^{s}, q^{2 t-s} ; q^{2 t}\right)_{\infty}^{3}:=\sum_{n=0}^{\infty} b_{r, s, t}(n) q^{n}
\end{aligned}
$$

where $t \geq 5$ is a prime, $r, s$ are positive integers and $r<t, s \neq t$.

Following the same line of proving (1.5)-(1.8), we can also obtain

$$
\begin{gathered}
b_{1,4,5}(5 n+3)=b_{2,2,5}(5 n+4)=0, \\
a_{1,1,7}(7 n+2)=a_{1,1,7}(7 n+5)=0, \\
b_{1,5,7}(7 n+3)=b_{1,5,7}(7 n+4)=0, \\
a_{3,3,7}(7 n+5)=a_{3,3,7}(7 n+6)=0, \\
b_{3,1,7}(7 n+3)=b_{3,1,7}(7 n+5)=0, \\
a_{4,6,11}(11 n+3)=b_{4,2,11}(11 n+3)=0, \\
a_{5,2,11}(11 n+1)=b_{5,8,11}(11 n+4)=0 .
\end{gathered}
$$

There are other equalities similar to (3.3)-(3.9) for $t=7$ or 11 . However, there are no similar results for $t=13$ or 17 . It is natural to ask whether or not there exists a criterion which can be used for searching for vanishing coefficients of the arithmetic progressions in $a_{r, s, t}(n)$ and $b_{r, s, t}(n)$.

On the other hand, the product representation of the Rogers-Ramanujan fractions is given by [7, Eq. (16.2.1)]:

$$
R(q)=\frac{\left(q, q^{4} ; q^{5}\right)_{\infty}}{\left(q^{2}, q^{3} ; q^{5}\right)_{\infty}}=\sum_{n=0}^{\infty} u(n) q^{n} .
$$

Richmond and Szekeres [9] also examined asymptotically the power series coefficients of a large class of infinite products including (3.10). They proved that, for sufficiently large $n$,

$$
u(5 n), u(5 n+2)>0, \quad \text { and } \quad u(5 n+1), u(5 n+3), u(5 n+4)<0 .
$$

A similar result was also obtained for the coefficients of $1 / R(q)$. 
In 1981, Andrews [3] used partition-theoretic interpretations of these coefficients, to prove (3.11) holds for all $n \geq 0$, except that $u(3)=u(8)=u(13)=u(23)=0$. Hirschhorn [6] later provided an elementary proof of (3.11) using only the quintuple product identity.

With the aid of computer, the signs of coefficients in $q$-series (3.1) and (3.2) appear to be periodic from some large $n$ for $t=5,7$, and 11. For example,

$$
\begin{array}{rll}
b_{1}(5 n), b_{1}(5 n+2), b_{1}(5 n+3)>0, & \text { and } & b_{1}(5 n+4)<0, \\
a(10 n), a(10 n+3), a(10+6)>0, & \text { and } & a(10 n+1), a(10 n+5), a(10 n+8)<0, \\
a_{1,1,7}(7 n+1), a_{1,1,7}(7 n+6)>0, & \text { and } & a_{1,1,7}(7 n+3), a_{1,1,7}(7 n+4)<0, \\
b_{3,3,11}(22 n+2)>0, & \text { and } & b_{3,3,11}(22 n+13)<0 .
\end{array}
$$

However, it is unclear how these inequalities could be proved by $q$-series.

Furthermore, the numerical evidence suggests the following conjecture.

Conjecture 3.1. For given $r, s$, and $t$, the signs of $a_{r, s, t}(n)$ 's and $b_{r, s, t}(n)$ 's are periodic with period $t$ or $2 t$ from sufficiently large $n$.

\section{ACKNOWLEDGEMENT}

The author is indebted to Shishuo Fu for his helpful comments on a preliminary version

of this paper. The author would like to acknowledge the referee for his/her careful reading and helpful comments on an earlier version of the paper. This work was supported by the National Natural Science Foundation of China (No. 11501061) and the Fundamental Research Funds for the Central Universities (No. 2018CDXYST0024).

\section{REFERENCES}

[1] K. Alladi and B. Gordon, Vanishing coefficients in the expansion of products of Rogers-Ramanujan type, Proc. Rademacher Centenary Conference, (G. E. Andrews and D. Bressoud, Eds.), Contemp. Math. 166 (1994), 129-139. 1

[2] G. E. Andrews and D. M. Bressoud, Vanishing coefficients in infinite product expansions, J. Austral. Math. Soc. Ser. A 27 (1979), no. 2, 199-202. 1

[3] G. E. Andrews, Ramanujan's "lost" notebook III. The Rogers-Ramanujan continued fraction, Adv. Math. 41 (1981), 186-208. 11

[4] B. C. Berndt, Ramanujan's Notebooks, Part III, Springer-Verlag, New York, 1991. 3

[5] M. D. Hirschhorn, Two remarkable $q$-series expansions, Ramanujan J., in press. 1, 4

[6] M. D. Hirschhorn, On the expansion of Ramanujan's continued fraction, Ramanujan J., 5 (1998), 521-527. 11

[7] M. D. Hirschhorn, The Power of q, Developments in Mathematics Vol. 49, Springer 2017. 3, 10

[8] J. McLaughlin, Further results on vanishing coefficients in infinite product expansions, J. Austral. Math. Soc. Ser. A 98 (2015), 69-77. 1

[9] B. Richmond and G. Szekeres, The Taylor coefficients of certain infinite products, Acta Sci. Math. (Szeged) 40 (1978), no. 3-4, 347-369. 1, 10

(Dazhao Tang) College of Mathematics and Statistics, Chongqing University, Huxi CamPus LD206, Chongqing 401331, P.R. China

E-mail address: dazhaotang@sina.com 